\title{
Performance Evaluation of Proximity Services and Wi-Fi for Public Safety Mission Critical Voice Application
}

\author{
Wesley D. Garey (D, Yishen Sun, and Richard A. Rouil \\ Wireless Networks Division, National Institute of Standards and Technology, Gaithersburg, MD 20899, USA \\ Correspondence should be addressed to Wesley D. Garey; wesley.garey@nist.gov
}

Received 12 December 2019; Accepted 29 April 2020; Published 21 May 2020

Academic Editor: Maximilian Matthé

Copyright (c) 2020 Wesley D. Garey et al. This is an open access article distributed under the Creative Commons Attribution License, which permits unrestricted use, distribution, and reproduction in any medium, provided the original work is properly cited.

\begin{abstract}
Proximity Services (ProSe) and Wi-Fi are two promising technologies that may provide support for Mission Critical Voice (MCV) applications in remote and rural areas by enabling Device-to-Device (D2D) communication. In this paper, several performance metrics of ProSe and Wi-Fi are evaluated and compared side-by-side under various configurations. The ns-3 simulation results show that ProSe outperforms Wi-Fi in terms of coverage range and access time with a medium traffic load, while Wi-Fi has a shorter access time under a light traffic load. In addition, with various user densities, ProSe offers better coverage range and access time a majority of the time. The evaluation in this paper provides insights to first responders on what to expect with either technology and how to improve the performance by adjusting different system parameters.
\end{abstract}

\section{Introduction}

Communication is vital for first responders in mission critical situations as it may be the difference between life and death. While there may exist a vast network infrastructure intended to provide first responders with broadband wireless access, it may not always be available or the most viable option at any given point in time. Regardless, the Mission Critical Voice (MCV) service should be resilient and available and provide its required function when necessary, even in rural or remote areas. In such a situation, first responders may use Device-to-Device (D2D) communication to stay connected. With D2D, first responders are able to maintain connectivity over a direct link between members of a group, allowing them to communicate with peers in their local vicinity without the intervention of a centralized entity. How reliable that link is will depend heavily on the underlying technology at hand.

Proximity Services (ProSe) is a feature of Long-Term Evolution (LTE) that was first introduced by the $3^{\text {rd }}$ Generation Partnership Project (3GPP) in 2014 and is used to provide $\mathrm{D} 2 \mathrm{D}$ communication. This technology provides synchronization, discovery, and communication services for local User Equipment (UE). Different modes of operation have been defined allowing public safety devices to communicate when both in-coverage and out-of-coverage of an LTE network. When out of coverage, UEs rely on preconfigured information to determine which resources to use [1]. This mode of operation is referred to as mode 2. While ProSe is deemed to be promising to support first responders in rural and remote areas, this LTE feature has yet to be widely adopted or implemented like its counterpart, Wi-Fi. Wi-Fi is an Institute of Electrical and Electronics Engineers (IEEE) standard that can be used in ad hoc mode to also provide D2D communication. While there are many different flavors of $\mathrm{Wi}-\mathrm{Fi}$, in this paper the term $\mathrm{Wi}-\mathrm{Fi}$ is used to refer to the commercially available IEEE $802.11 \mathrm{~g}$ standard that was ratified in 2003 [2]. Wi-Fi is implemented in many devices today and is able to provide high-throughput and shortrange wireless communication. As is described in [3] and other literature, LTE and Wi-Fi are both promising candidates for first responders, capable of providing communication during incidents in rural and remote areas where network coverage may be limited. Even though quite some effort has been put into investigating the performance of ProSe and Wi-Fi, only a few works compare them with 
another technology, while even less consider them for use by public safety.

Works [4-8] focus their efforts on characterizing the performance of ProSe alone. In [4], a thorough investigation is conducted that studies Block Error Rate (BLER) performance for the various ProSe channels at the physical layer. This work is later extended in [5] to determine the link capacity and coverage range of ProSe. While [5] considers many different deployment scenarios and the impact of various system parameters, it does not take into account the different applications that will use ProSe, e.g., mission critical applications. A mathematical model is presented in [6] that characterizes the likelihood of a UE receiving a message when multiple UEs are sharing the same channel. While [6] is thorough, it assumes any collisions that occur result in the loss of a message and does not consider the Signal-to-InterferencePlus-Noise Ratio (SINR) of the received message that could potentially be recovered, making it a very conservative approach. In [7], an investigation of Mission Critical Pushto-Talk (MCPTT) Access Time is performed to analyze how soon a first responder should expect to gain access to the channel after a Push-to-Talk (PTT) request is made. Again, while [7] is extensive and provides an analytical model to give a general idea of MCPTT performance over ProSe, the analysis only takes into account collisions that occur from application control messages which are negligible when compared to the amount of data messages that can be produced by the MCPTT application. A software-based implementation of ProSe, developed in ns-3, is presented in [8]. This is the same model that is used in $[6,7]$ that will also be used in this paper.

Among the numerous research papers that provide a performance evaluation of $\mathrm{Wi}$-Fi, only a small portion of papers dealt with public safety use cases and/or voice applicationrelated performance metrics. While investigating the performance of mesh networks to be used for public safety, [9] also highlights the shortcomings of Wi-Fi. Although the paper suggests the need and the prominent issues, such as capacity and interference, for deploying wireless networks in small towns and rural areas, the study itself is very limited since it only considers the throughput of unknown sources. In [10], an analytical model is developed that characterizes the Voice Over IP (VoIP) call capacity of single-hop and multihop Wi-Fi networks using various codecs. An overview of Bluetooth and Wi-Fi is provided in [11], which describes how the protocols operate, their main features, and how they compare in terms of spectrum, power requirements, security, etc. In [12], a log-distance model is created to predict bit rate versus distance for Wi-Fi using physical devices in a remote parking lot. While deriving this model, it is also determined that $\mathrm{Wi}-\mathrm{Fi}$ can deliver close to $5 \mathrm{Mbits} / \mathrm{s}$ at $100 \mathrm{~m}$. The Wi-Fi error model in $\mathrm{ns}-3$ is validated in [13], which describes the SINR of the Wi-Fi model in ns-3 being within $1 \mathrm{~dB}$ of the Wi-Fi devices used with a wireless channel emulator. In [14], an extensive analysis is performed using simulation to investigate the performance of $802.11 \mathrm{~b}$ ad hoc networks. Both [14] and [15] highlight the shortcomings of Wi-Fi networks and describe the presence of "hidden stations," which we will refer to in Section 5.2.
While the above and others analyze the performance metrics (e.g., range, link capacity, and access time) of Wi-Fi and ProSe separately, we do not know of any published papers that compare the two under similar assumptions and in a comparable environment. Thus, the question that still remains is as follows: How does ProSe compare to WiFi with respect to public safety needs when it comes to providing a reliable link for voice communication without the network infrastructure? To address this issue, we study the impact that each technology has on various important performance metrics. First, in Section 2, the simulation tools and channel models used to perform the investigation are introduced. Then, in Section 3, we study the impact that the system load has on the 3GPP-defined MCPTT access time of an MCV application, which indicates how quickly a user may access the channel. In Section 4, MCV coverage probability is defined and is used to measure the range that is achievable by both technologies using different configurations with regard to the MCV application. In Section 5, the user density analysis will be conducted for a more complete and comprehensive study of the technologies. Lastly, we will provide insights and conclusions to the analyses that were performed.

\section{Model Overview}

In this paper, we consider various scenarios in which at least two UEs of the same group are communicating after being deployed in a disk-shaped area. The analysis starts with one pair of UEs in the system as shown in Figure 1. In Sections 3 and 5, more users are considered to study the impact of the traffic load and/or user density. These additional UEs are deployed simply to introduce more traffic, so even as these additional UEs are introduced, the statistics generated for each sample are still taken only from a designated pair of UEs.

The MCPTT application model we use in the simulation assumes voice packets are generated by a high-quality Adaptive Multirate Wideband (AMR-WB) encoder, G.722.2, as described in [16]. The vocoder generates 60 byte voice packets every $20 \mathrm{~ms}$ when the MCV user is actively talking. This means that for voice communication the demand is $24 \mathrm{kbits} / \mathrm{s}$ at the application level, but at the link layer, the source data rate is even higher after taking into account the various headers that have to be added to the 60 byte voice packets depending on the wireless communication technology. Note that for each analysis, it is assumed that enough resources are made available by both technologies so that the bandwidth of the link is large enough to accommodate all possible traffic with no fragmentation. We also assume for both ProSe and Wi-Fi that all devices use the same configuration. The headers that are considered for both technologies are shown in Table 1 and Figure 1. Consequently, a wireless D2D link should provide at least $44 \mathrm{kbits} / \mathrm{s}$ or $42.4 \mathrm{kbits} / \mathrm{s}$ data rate when using Wi-Fi or ProSe, respectively, in order to support the MCPTT voice application. In addition to voice, the MCPTT floor control [17] and MCPTT call control protocols [18] are implemented to support MCPTT operations. The control messages generated by these protocols also require resources even though the amount of resources that are needed is not as significant as the voice traffic. 


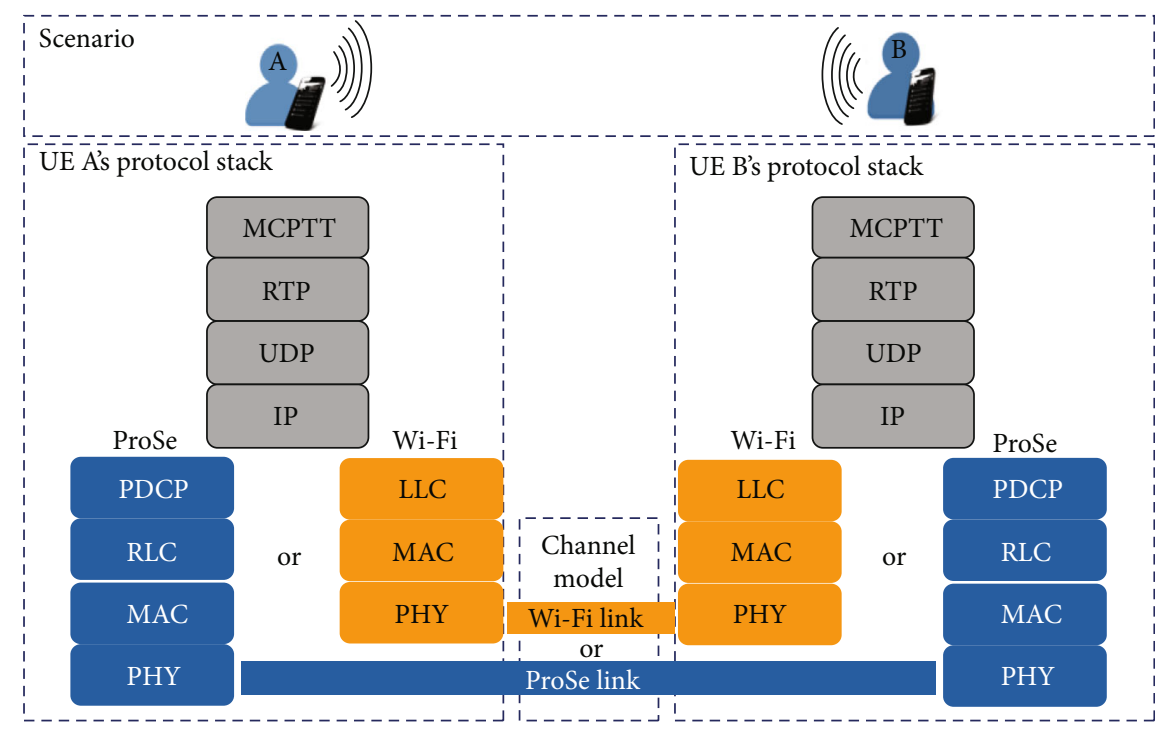

FIgURe 1: Components in the model.

TABLE 1: The sizes of the protocol headers.

\begin{tabular}{lcc}
\hline Header & Size (bytes) & Technology \\
\hline Real-Time Transport Protocol (RTP) & 14 & Both \\
User Datagram Protocol (UDP) & 8 & Both \\
Internet Protocol (IP) & 20 & Both \\
Packet Data Convergence Protocol & 2 & ProSe \\
(PDCP) & 2 & ProSe \\
Radio Link Control (RLC) & 8 & Wi-Fi \\
Logical Link Control (LLC) & & \\
\hline
\end{tabular}

The propagation models used in our simulations for both ProSe and Wi-Fi are the simple Friis model as specified in [19] and the more comprehensive models from Wang and Rouil in $[4,5]$. In Section 3, the Friis model is used when analyzing the impact of the traffic load on access time. This enables us to focus solely on the transmission errors caused by collisions and the half duplex effect which are identified in [7] as this is the main consequence of increased traffic load. When evaluating the impact of the range in Section 4 and user density in Section 5, Wang and Rouil's model is used, which takes path loss, shadowing, and interference into account. It is worth mentioning that we use Wang and Rouil's model instead of the default log-distance model [13] for $\mathrm{Wi}-\mathrm{Fi}$, simply to make it possible for an apples-toapples comparison of ProSe and Wi-Fi. It is also worth mentioning that the appropriate frequency values are configured as the input parameter for both propagation models: $793 \mathrm{MHz}$ (Band 14 uplink frequency) for ProSe and 2.407 GHz (802.11 b/g/n) for Wi-Fi.

To support off-network mode MCPTT functionalities, D2D links need to be established between UEs. Therefore, ProSe UEs are operated in mode 2 [20], while Wi-Fi UEs are operated in ad hoc mode [21]. With these modes of operation, both technologies provide the necessary functions for
UEs to communicate directly with one another so neither relies on an underlying infrastructure of base stations or access points. In each scenario simulated, all UEs in the system use the same technology to communicate. That is, the system is composed of all ProSe UEs or all Wi-Fi UEs, not both simultaneously.

Even though the results presented in Sections 3 and 5 focus on one set of ProSe parameters and one set of Wi-Fi parameters, we have studied other configuration values already and observed similar trends. A variety of system configurations are discussed in Section 4 to study the effect that the system parameters have on coverage range.

\section{Impact of Traffic Load on Access Time}

In this section, MCPTT access time is selected as the first performance metric to compare ProSe with Wi-Fi. This is because access time is a very important Key Performance Indicator (KPI) for first responders [7]. In the corresponding 3GPP standards [22], the MCPTT access time is defined as follows.

Definition 1. (MCPTT access time). The amount of time from when the user requests to talk until the time the user gets an indication that he/she may talk.

In the remainder of this section, the major assumptions for the corresponding analyses are listed in Section 3.1, the MCPTT access time obtained over ProSe and Wi-Fi is compared for a single pair of UEs in Section 3.2, and the MCPTT access time obtained over ProSe and Wi-Fi with additional users is compared in Section 3.3.

3.1. Assumptions. The following scenarios assume that first responders have already joined an active call, which means that once a first responder has been granted the floor (i.e., has obtained permission to speak), he/she may start speaking and voice packets (RTP) can be sent. In 


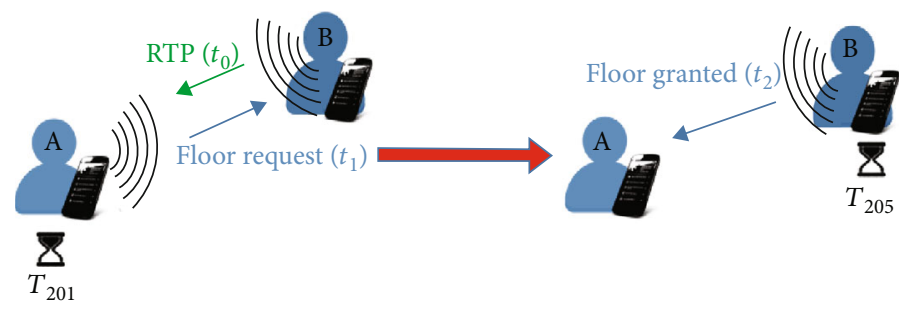

FIgURE 2: Preemptive floor request scenario with voice traffic.

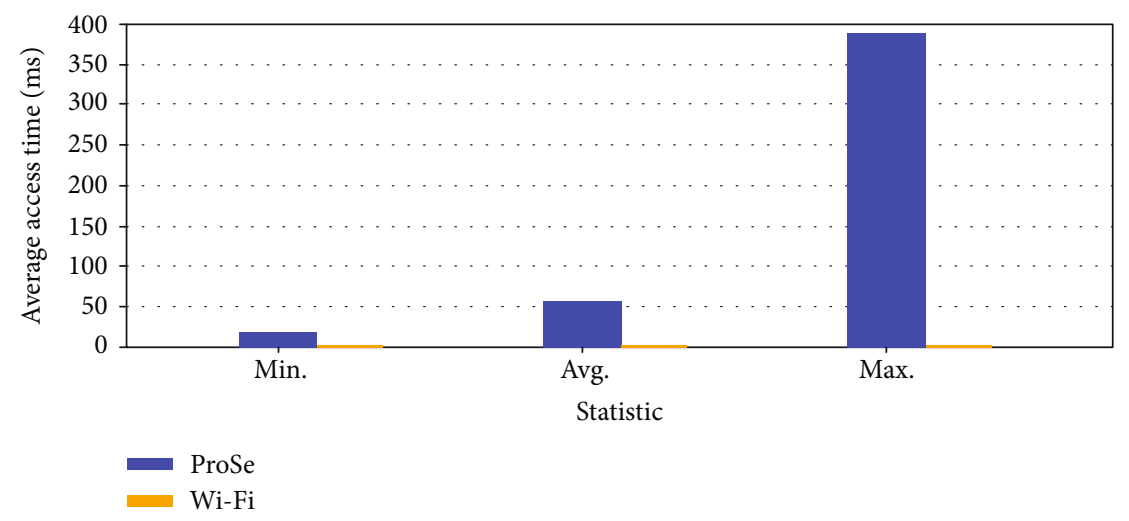

FIgURe 3: Baseline MCPTT access time with error bars that represent the $95 \%$ confidence interval.

addition, to focus on the impact of the traffic load on access time, we assume a floor participant UE, $U E_{A}$, sends a preemptive floor request and thus the floor arbitrator $U E, U E_{B}$, has to grant the floor to $U E_{A}$ without further delay. A more detailed explanation of the procedure can be found in Section 7 of [17] or a more general description can be found in procedure F2.1 of [7]. Note that voice traffic and system load are not considered in [7] but their significance will become clear in Sections 3.3 and 5.2. In addition, any collisions that occur result in the loss of both packets, which is the same assumption as in $[6,7]$.

The MCV application on a UE will be generating voice packets at $24 \mathrm{kbits} / \mathrm{s}$ and control messages from the call control and floor control protocols. In the simulation, we assume a ProSe sidelink period to be $40 \mathrm{~ms}$. Thus, the value of $T_{201}$ (the floor request retransmission timer) is set to $40 \mathrm{~ms}$ and the value of $T_{205}$ (the floor granted retransmission timer) is $80 \mathrm{~ms}$ based on the 3GPP recommended default values [17]. Note that no $T_{201}$ or $T_{205}$ values are given for $\mathrm{Wi}-\mathrm{Fi}$ since the MCPTT protocol is specified to work over ProSe and does not consider Wi-Fi. To address this, we simply use the same values for both ProSe and Wi-Fi. In addition, the $6 \mathrm{Mbits} / \mathrm{s}$ data rate mode is used for $\mathrm{Wi}-\mathrm{Fi}$, while the Modulation and Coding Scheme $(\mathrm{MCS})=18$, number of Physical Resource Blocks (PRBs) $N_{\mathrm{PRB}}=4$, and $k_{\mathrm{TRP}}=2$, which denotes the number of transmissions opportunities in a Time Resource Pattern (TRP) for ProSe. More details are given in Section 4 that will explain why the above system parameters are selected for ProSe and Wi-Fi to support the applications' demand.
3.2. Single-Pair Analysis. The analysis starts with the basic scenario of a single-pair case as shown in Figure 2. The simulation results shown in Figure 3 compare the access time statistics of ProSe vs. Wi-Fi based on 100000 samples. The MCPTT access time (in milliseconds) is on the $y$-axis, with the recorded statistics (minimum, average, and maximum) on the $x$-axis. Even though the confidence interval at $95 \%$ is included in every figure with the average access time, those error bars are not visible in Figures 3 and 4(a) since the confidence interval for both technologies is less than $1 \mathrm{~ms}$. As can be seen in Figure 3, the access times for Wi-Fi are much smaller than they are for ProSe, with $\mathrm{Wi}$-Fi providing a minimum, maximum, and average access time less than $5 \mathrm{~ms}$. This difference in access time is attributed to the differences in each technology's medium access control schemes. For ProSe, the sidelink period behaves like a time window that comes around to all UEs periodically, so there is a restriction on the time frame in which a UE may transmit even if the channel is idle. This results in a scheduling delay that puts a lower bound on how soon after a packet is ready to be sent that it is actually transmitted. At the same time, this window also puts an upper bound on how long a packet will be queued for transmission, assuming the traffic demand does not exceed available radio resources. This is because as long as the packet is ready to be transmitted before the scheduling cutoff time of the next sidelink period, it will be transmitted in the next sidelink period. This behavior is described with great detail in Section 2 of [7]. On the contrary, Wi-Fi UEs can transmit almost instantly, as a UE may access the channel as soon as a packet is ready to be transmitted 


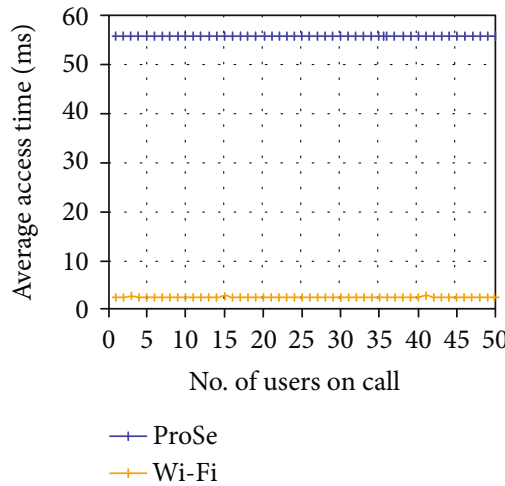

(a)

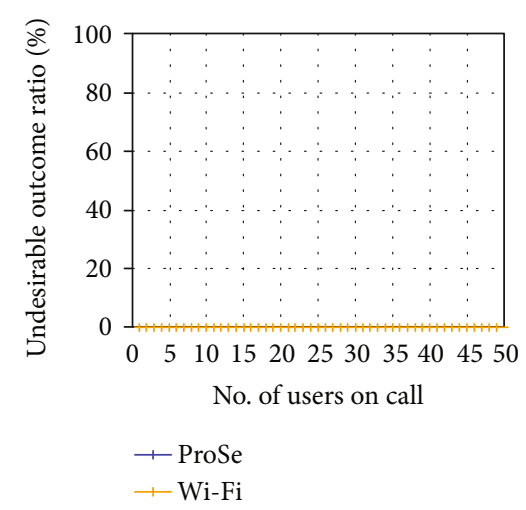

(b)

Figure 4: Performance metrics for additional users in a single call. (a) MCPTT access time. The error bars represent the 95\% confidence interval. (b) Undesirable outcome ratio.

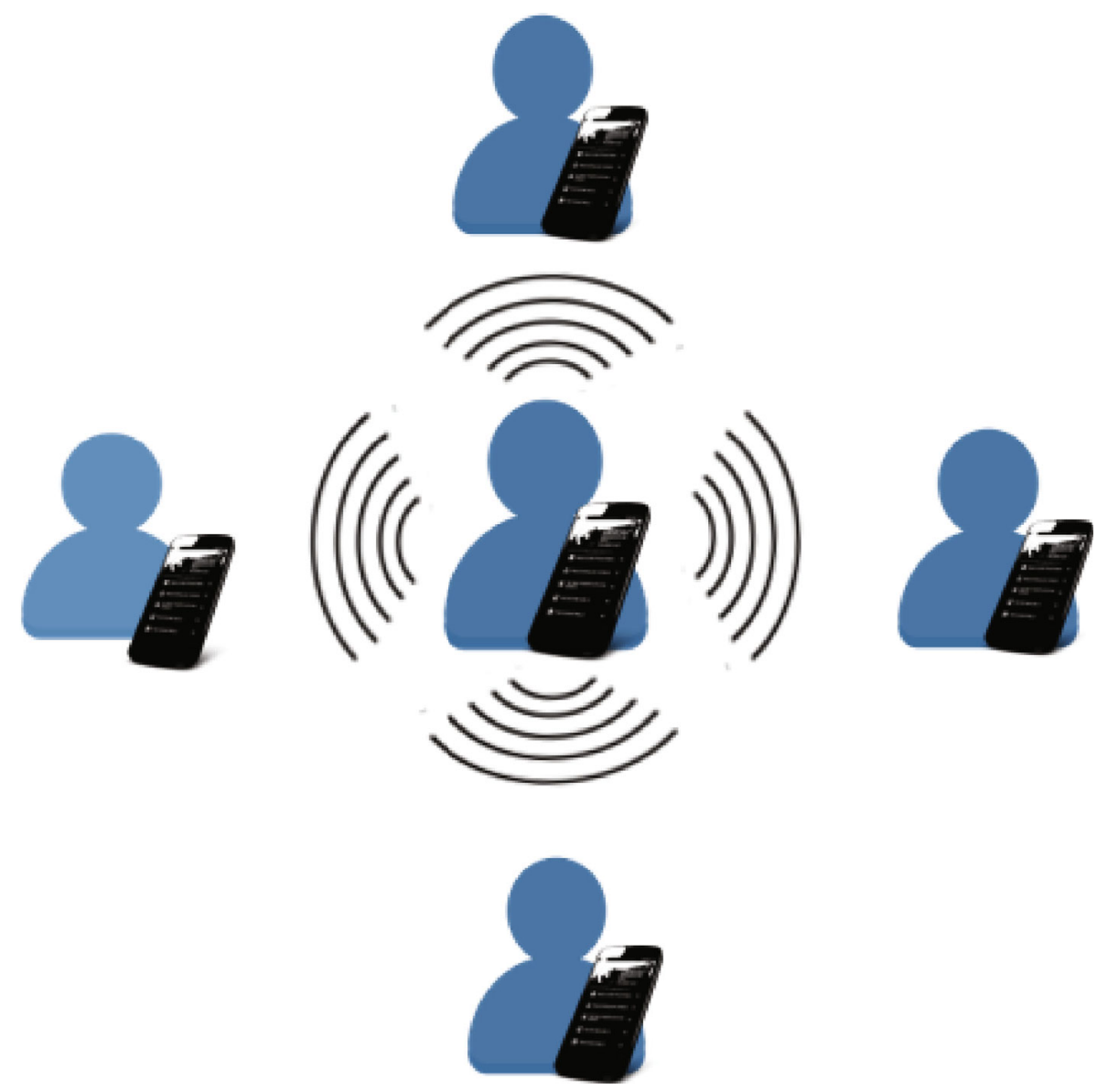

Figure 5: Multiple users on a single call.

as long as no other UE is transmitting. This is made possible by Wi-Fi's Distributed Control Function (DCF), which utilizes Carrier-Sense Multiple Access with Collision Avoidance (CSMA/CA) [2] to mitigate collisions.

3.3. Multipair Analysis. In this section, we will compare how additional UEs may impact the access time performance of
MCPTT over ProSe vs. that of MCPTT over Wi-Fi. Two sets of scenarios are considered: first, we will evaluate the impact of more UEs on a single call (Figure 5); then, we will evaluate the impact of additional UEs on separate calls (Figure 6). For each call, we assume that only one UE is actively talking, meaning that in each call there is a single UE with a single application that has a demand of $24 \mathrm{kbits} / \mathrm{s}$. 

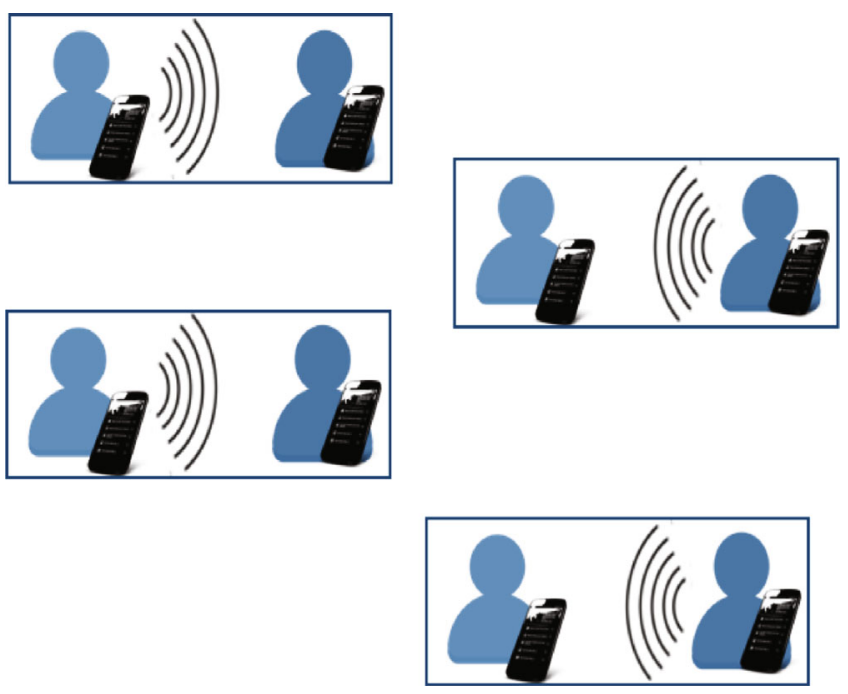

FIGURE 6: Multiple users on separate calls.

As can be seen in Figure 4(a), when adding more UEs to a single call, the impact on access time seems to be negligible for both technologies. This is because the amount of additional traffic introduced in this scenario is minimal due to the fact that only a single UE, i.e., the floor arbitrator, is ever transmitting voice packets at a time. The only additional traffic that is observed in this scenario is the periodic transmission of a "Group Call Announcement" message that should only be sent by a single UE about every 10 seconds. We also see a similar trend in Figure 4(b), the ratio of undesirable outcomes. Coined in [7], this ratio is tightly coupled with the access time to illuminate the number of instances in which the user receives an indication that he/she may begin speaking and the UE is able to transmit voice packets, but the floor granted message was never received by $U E_{A}$. This can happen if the floor arbitrator never receives the floor request from $U E_{A}$, or if $U E_{A}$ never receives the floor granted message from the floor arbitrator. In either case, it is possible that members of the group will hear only part or none of the requesting user's transmission.

From Figure 7(a), we can see that the additional UEs on separate calls impact the access time performance for both technologies more noticeably. As more separate calls are introduced, the access time increases from a few milliseconds to several hundred milliseconds, with maximums (not shown in graphs) exceeding 1 second. This is due primarily to the fact that in each additional call one UE is generating voice packets at $24 \mathrm{kbits} / \mathrm{s}$. Observe that once 19 separate calls are active in the system, there is a crossover point where $\mathrm{Wi}-\mathrm{Fi}$ no longer outperforms ProSe and begins to perform worse. The trend of the relative performance of each technology is a consequence of the medium access control methods mentioned earlier. Although the DCF made it possible for Wi-Fi UEs to instantly access the channel when there was less traffic, this method does not scale well when more UEs in the system are actively transmitting. This is because, as more traffic is introduced, it is more likely that a signal will occupy the Wi-Fi channel. Thus, the back-off algorithm will delay the transmission of the control messages sent by $U E_{A}$ and
$U E_{B}$ that must be exchanged in order for access to be requested and then granted. On the contrary, ProSe randomizes the selection of radio resources for transmission to minimize potential collisions, instead of sensing the channel. As explained in Section 3.2 already, this scheduling method puts an upper bound on how long a packet will be queued for transmission, assuming the traffic demand does not exceed available radio resources. Therefore, the access time of MCPTT over ProSe scales better when more UEs are introduced into the system: as more sidelink resources are occupied by more UEs, the access time increases gradually, instead of rapidly as for Wi-Fi. Once the channel is so saturated, i.e., there are around 25 group calls with an active talker, we see that the access time begins to decrease for $\mathrm{Wi}-\mathrm{Fi}$ and the rate at which the access time is increasing for ProSe begin to fall. This does not mean that the performance is stabilizing or improving, but rather that the occurrence of undesirable outcomes is increasing. Figure 7(b) displays this increase. Therefore, we can see that Wi-Fi outperforms ProSe when the number of active calls (i.e., amount of traffic) is small, but as more calls exist, eventually ProSe outperforms Wi-Fi. When the number of separate calls is so large, although the performance of both technologies is similar, neither is satisfactory due to the high ratio of undesirable outcomes.

In order to reduce the ratio of undesirable outcomes when there are a large number of active calls, we studied the pros and cons of adjusting $T_{201}$. For the 2 UE scenario of MCPTT over ProSe, [7] already showed that the larger the $T_{201}$ value, the less likely a floor participant will assume the floor arbitrator role prematurely, and thus, the ratio of undesirable outcome occurrence may be smaller. For the comparison of Wi-Fi vs. ProSe and for the multiple UE scenario, the impact of the $T_{201}$ configuration is shown in Figures $8(\mathrm{a})$ and $8(\mathrm{~b})$. The number of separate calls is still on the $x$-axis, and either the average access time or undesirable outcome ratio is on the $y$-axis. There are 3 curves for each technology and each of them corresponds to a different $T_{201}$ value. Consistent with the findings in [7], we observe in Figure $8(\mathrm{~b})$ that by increasing the configuration value of timer $T_{201}$, we can mitigate the occurrence of undesirable outcomes but not without incurring an increase in access time for both technologies, which can be seen in Figure 8(a).

\section{Impact of Range on Coverage Probability}

In this section, we define and analyze a coverage probability that applies to the MCV application. Moreover, this analysis compares the range of ProSe and Wi-Fi based on a set of constraints that are imposed on the loss, delay, and data rate of our MCV application model.

4.1. Assumptions. In this analysis, we simulate a scenario with two UEs that are positioned $x$ meters apart from each other with a wireless link provided by either ProSe or Wi-Fi. An MCV application transmits voice packets from one UE to the other for a total of 20 seconds. The loss, average delay, and throughput are derived from the resulting traces and are later used to determine the coverage probability. 2000 


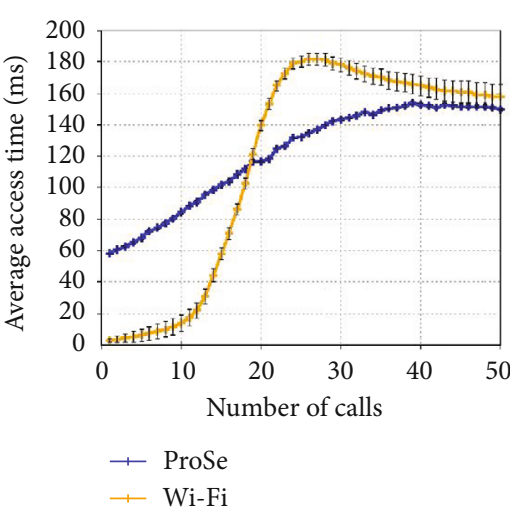

(a)

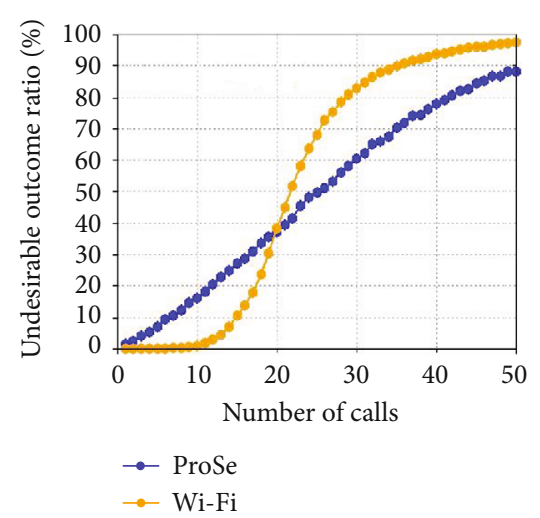

(b)

FIgURE 7: Performance metrics for additional calls. (a) MCPTT access time. The error bars represent the 95\% confidence interval. (b) Ratio of undesirable outcomes.

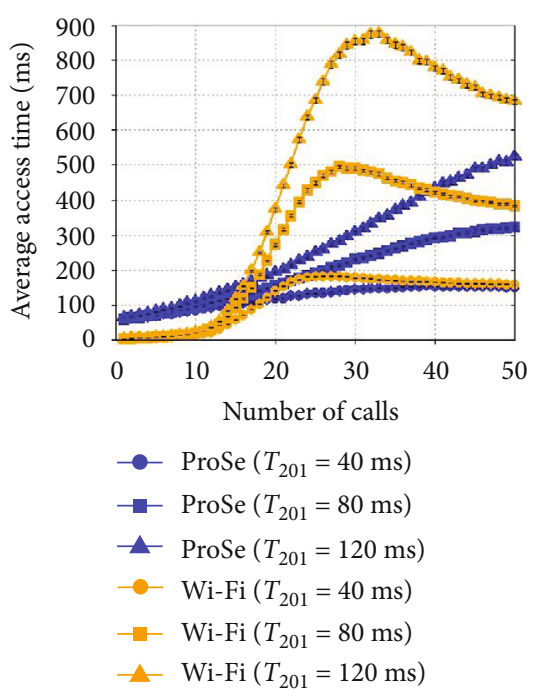

(a)

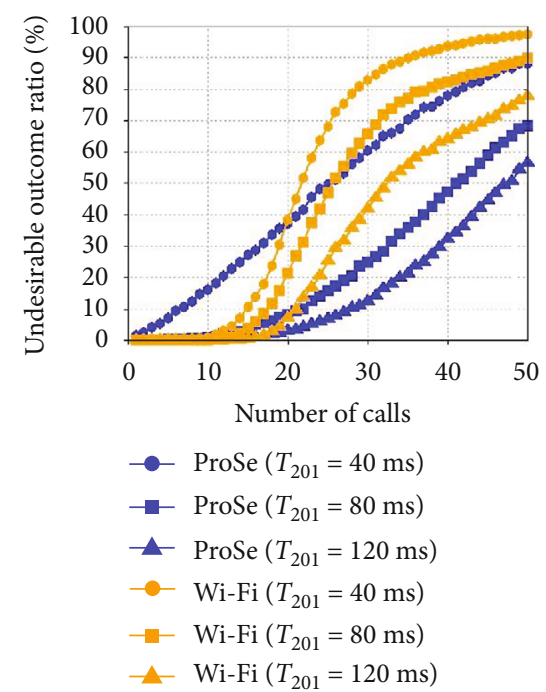

(b)

Figure 8: Performance metrics for additional calls using various $T_{201}$ values. (a) MCPTT access time. The error bars represent the $95 \%$ confidence interval. (b) Undesirable outcome ratio.

simulation runs are conducted for each distance and system configuration.

According to [23], the requirements for MCPTT speech are a packet delay of $75 \mathrm{~ms}$ or less and a packet loss ratio of $1 \%$ or less. In [16], the data rate for the G.722.2 AMR-WB encoder and decoder is $23.85 \mathrm{kbits} / \mathrm{s}$ so we assume that the data rate must be at least this much. Thus, for any simulation at distance $x$, if all three metrics (delay, throughput, and loss ratio) meet the minimum requirements, the UE is determined to be covered; otherwise, the UE is not covered. Therefore, the MCV coverage probability is defined as follows.

Definition 2. (MCV coverage probability). The ratio of samples for which the packet loss is no more than $1 \%$, the average packet delay is no more than $75 \mathrm{~ms}$, and the resulting data rate is no less than $23.85 \mathrm{kbits} / \mathrm{s}$ at the conclusion of the transmission period for a pair of UEs.
The system parameter values used for the simulations in this section are selected such that the demand of the MCV application $(24 \mathrm{kbits} / \mathrm{s})$ can be met. For Wi-Fi, the following data rates are simulated because they can support the MCV application demand: 6, 24, and 54 Mbits/s. For ProSe, Table 2 summarizes the various sets of system parameter values that are simulated in this section.

To be more specific, for a typical ProSe configuration with a $40 \mathrm{~ms}$ sidelink period and $N_{\mathrm{TRP}}=8$, the valid $k_{\mathrm{TRP}}$ values are $1,2,4$, and 8 , where $N_{\text {TRP }}$ is the number of $m s$ that is available for transmission and $k_{\mathrm{TRP}}$ is how many of those $N_{\text {TRP }} m s$ will be used for a transmission. The remaining 40 $-N_{\mathrm{TRP}} m s$ are a repetition of the first $N_{\mathrm{TRP}} m s$. Thus, for a given source traffic rate that requires $b$ bits to be transported every sidelink period, which in our case, $b=1696$ (two 106 byte voice packets), we can select an MCS and number of PRBs, $N_{\mathrm{PRB}}$, so that the size of a single Transport Block 
TABle 2: List of ProSe configurations that are considered.

\begin{tabular}{|c|c|c|c|c|c|c|c|c|c|c|}
\hline \multirow[b]{2}{*}{$k_{\mathrm{TRP}}$} & \multirow[b]{2}{*}{$P_{\mathrm{TB}}$} & \multicolumn{3}{|c|}{ Low } & \multicolumn{3}{|c|}{ Medium } & \multicolumn{3}{|c|}{ High } \\
\hline & & MCS & $N_{\mathrm{PRB}}$ & $N_{\mathrm{TB}}$ & MCS & $N_{\mathrm{PRB}}$ & $N_{\mathrm{TB}}$ & MCS & $N_{\mathrm{PRB}}$ & $N_{\mathrm{TB}}$ \\
\hline 2 & 848 & 0 & 32 & 872 & 10 & 5 & 872 & 20 & 3 & 1384 \\
\hline 8 & 212 & 0 & 9 & 224 & 7 & 2 & 224 & 14 & 1 & 256 \\
\hline
\end{tabular}

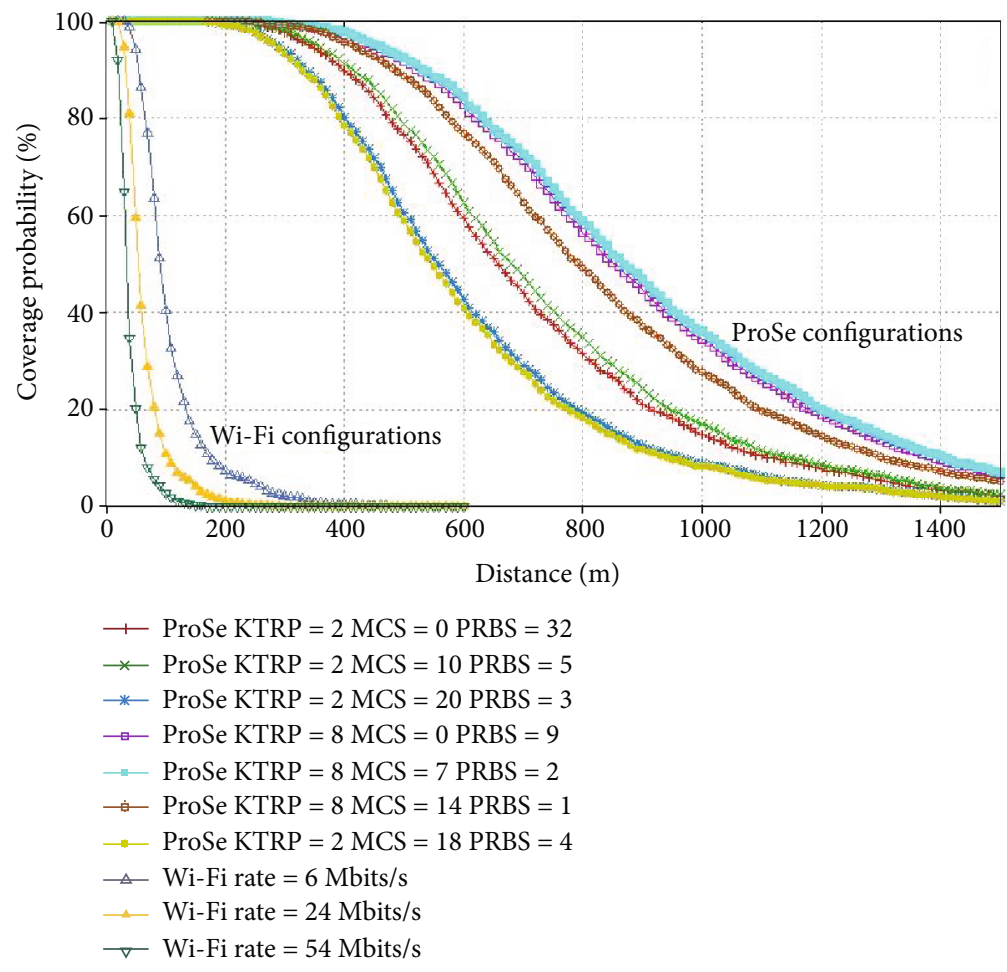

FIgURE 9: The baseline MCV coverage probability.

(TB), $N_{\mathrm{TB}}$, can transport $b$ bits. Put another way, the MCS and $N_{\mathrm{PRB}}$ are selected so that the range of feasible $N_{\text {TB }}$ values are key for determining MCS and $N_{\mathrm{PRB}}$ combinations. Table 7.1.7.2.1-1 in [24] maps an MCS and $N_{\mathrm{PRB}}$ selection to an $N_{\mathrm{TB}}$, which ultimately determines the maximum data rate that can be supported by ProSe. But since there exist multiple combinations of MCS and $N_{\mathrm{PRB}}$ that can support $N_{\mathrm{TB}}$, we vary the combinations by selecting a "low," "medium," and "high" MCS since, as will be shown later, this combination affects the MCV coverage probability.

4.2. Analysis. In Figure 9, we have the distance, $x$, on the $x$-axis and the measured MCV coverage probability on the $y$ -axis. Each line in Figure 9 corresponds to a technology with a specific configuration. Thus, "Wi-Fi Rate $=6 \mathrm{Mbits} / \mathrm{s}$ " corresponds to the MCV coverage probability obtained with a Wi-Fi link using the $6 \mathrm{Mbits} / \mathrm{s}$ data rate, and "ProSe KTRP $=2 \mathrm{MCS}=10 \mathrm{PRBS}=5$ " corresponds to the MCV coverage probability obtained with a ProSe link using $k_{\mathrm{TRP}}=2, \mathrm{MCS}=10$, and $N_{\mathrm{PRB}}=5$. This tells us that with a $6 \mathrm{Mbits} / \mathrm{s} \mathrm{Wi}-\mathrm{Fi}$ link, a 90\% MCV coverage probability can be maintained up until about $55 \mathrm{~m}$, while a ProSe link with
$k_{\mathrm{TRP}}=8, \mathrm{MCS}=7$, and $N_{\mathrm{PRB}}=2$ can achieve a $90 \%$ coverage probability up until about $530 \mathrm{~m}$. It is clear from Figure 9 that ProSe provides much greater coverage than $\mathrm{Wi}-\mathrm{Fi}$, and this difference could play a significant role in the success of first responder operations since natural disasters in rural areas can span tens of $\mathrm{km}^{2}$ [3].

As we mentioned before, different ProSe configurations also lead to different MCV coverage probabilities. For example, the higher the MCS, the lower the MCV coverage probability. This is because the data transmission with a higher MCS requires a higher SINR ratio at the receiver to decode the message successfully. For a similar reason of SINR, the larger the $N_{\mathrm{PRB}}$ is, the lower the MCV coverage probability. This is due to the fact that $N_{\mathrm{PRB}}$ determines how many symbols are available and thus directly affects the distribution of the transmit power. For instance, if $N_{\mathrm{PRB}}=1$ is used, then all the transmit power, $P_{\mathrm{TX}}$, will be concentrated into a single $\mathrm{PRB}$, whereas if $N_{\mathrm{PRB}}>1$, then the transmit power is distributed equally among. This means that as you increase $N_{\text {PRB }}$, you are effectively decreasing the received power of the TB which, consequently, leads to a lower SINR. In addition, a larger $k_{\mathrm{TRP}}$ may improve the MCV coverage probability. To 


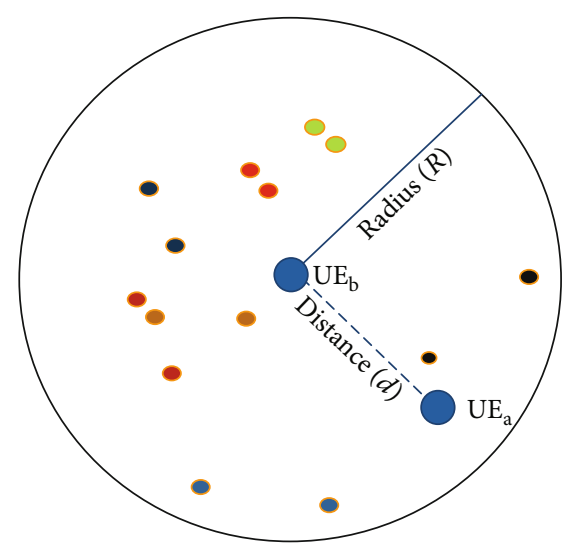

FIGURE 10: The user density topology.

be more specific, if we need to transmit a total of $b$ bits and $k_{\mathrm{TRP}}=1$, then all $b$ bits need to fit in a single TB. But if $k_{\mathrm{TRP}}$ $=2$, then each TB only needs to transport bits and thus a smaller $N_{\text {TB }}$ is required. This is significant because a smaller $N_{\text {TB }}$ can be achieved with a lower MCS and/or fewer PRBs, which translates to a lower SINR threshold to decode the message and/or an increase in the received power of the TB. The downside of using a larger $k_{\mathrm{TRP}}$ is that it may increase the likeliness of a collision, but in this analysis, only one user is ever transmitting at a time.

For $\mathrm{Wi}-\mathrm{Fi}$, it can be seen that the selected data rate plays a large role in the MCV coverage probability as the $6 \mathrm{Mbits} / \mathrm{s}$ data rate can achieve $90 \%$ beyond $50 \mathrm{~m}$ while the distance for $54 \mathrm{Mbits} / \mathrm{s}$ is just past $20 \mathrm{~m}$. This is because the selected data rate determines the key parameter MCS for transmission over the air. Similar to ProSe, a higher data rate is achieved with a higher MCS, but this ultimately sacrifices coverage.

\section{Impact of User Density on Access Time and Coverage Probability}

5.1. Assumptions. In this section, we combine the various aspects of Sections 3 and 4 for a complete study that extends beyond an idealistic channel or a single pair of UEs. The need to combine these aspects comes from the fact that in Section 3 we assume that all devices can communicate regardless of distance, while in Section 4 only a single voice application transmits data packets. Therefore, the analysis in this section considers the impact of user density which gives insight on how many active calls can be supported in a given area before the performance of each technology degrades.

The user density topology is depicted in Figure 10. Simulation scenarios and system configurations in this section are very similar to those in Section 3.3 as shown in Figure 6. However, in this analysis, packets are dropped based on the BLER models used in Section 4 since these models take noise into account that is introduced by interfering UEs. In this topology, $R$ is the radius of the area in which UEs are deployed, and $d$ is the maximum distance that can be between a pair of UEs on a call in the same group. Each dot in the circle represents a UE, and the color of the UE represents the call that the UE is a part of. $R$ is selected based on the criteria that devices at distance $R$ have a coverage probability less than $1 \%$, which means that devices at this distance will likely not interfere with each other. $d$ will be varied using a subset of values between the distances at which a 95\% coverage probability is no longer achievable for either technology. Given the results from Section 4 , we will use $R=800 \mathrm{~m}$ with $d \in\{40,120,200,280\} m$ for both technologies.

5.2. Analysis. The results of the user density analysis can be seen in Figures 11(a)-11(c). On the $x$-axis we have $d$, and the $y$-axis is for coverage probability in Figure 11(a), the average access time in Figure 11(b), and the ratio of undesirable outcomes in Figure 11(c), respectively. Different curves in the figure reflect a different number of UE pairs with an active talker in the system. For example, the series "ProSe (5 calls)" corresponds to a ProSe system with 5 additional groups and a maximum distance of $d$ between any pair of UEs on the same call.

Figure 11(a) shows that both technologies can maintain a coverage probability greater than $90 \%$ when $d=40 \mathrm{~m}$, even when there are 45 additional calls. As expected, the Wi-Fi performance is very similar to ProSe at first but eventually falls slightly below that of ProSe as more traffic is introduced. In Section 4, when there was no additional traffic, the coverage probability for a pair of UEs using Wi-Fi or ProSe was very close to $100 \%$. Therefore, when $d=40 \mathrm{~m}$, we can see from the slight decrease in the curves that the predominant factor affecting both ProSe and Wi-Fi is interference. However, as we increase $d$, we can see that ProSe performs better at first and then falls to a performance similar to that of $\mathrm{Wi}$ Fi. We also notice that Wi-Fi is almost always worse than ProSe for a given $d$ and does not appear to change much as more traffic is introduced. This is because Wi-Fi is impacted more by the distance between devices while ProSe is impacted more by the total number of devices when 40 $m<d \leq 280 \mathrm{~m}$. Observe that as $d$ increases from $40 \mathrm{~m}$ to $280 \mathrm{~m}$ the coverage probability for $\mathrm{Wi}-\mathrm{Fi}$, with just 5 additional calls, decreases from $99 \%$ to $29 \%$, but as the number of additional calls increases from 5 to 45 and $d=280 \mathrm{~m}$ the coverage probability only decreases from $29 \%$ to $23 \%$. On the other hand, for ProSe, as $d$ increases from $40 \mathrm{~m}$ to $280 \mathrm{~m}$ with 5 additional calls the coverage probability only decreases from $99 \%$ to $82 \%$, but as the number of calls increases from 5 to 45 when $d=280 \mathrm{~m}$ the coverage probability drops from $82 \%$ to $22 \%$. We can also see that the coverage probability decreases much faster for ProSe than for $\mathrm{Wi}-\mathrm{Fi}$ as $d$ increases with the number of additional calls. This is because more calls lead to more UEs, and a larger $d$ means a more sparse deployment of UEs. Thus, the amount of interference increases because it is more likely for a UE to be closer in proximity to the member of another call.

As we can see from the curves in Figure 11(b), the average access time for Wi-Fi increases much sooner than for ProSe. For example, when $d=40 \mathrm{~m}$, the average access time increases from $131.20 \mathrm{~ms}$ with 5 additional calls to $624.57 \mathrm{~ms}$ when there are 45 additional calls, while for ProSe 


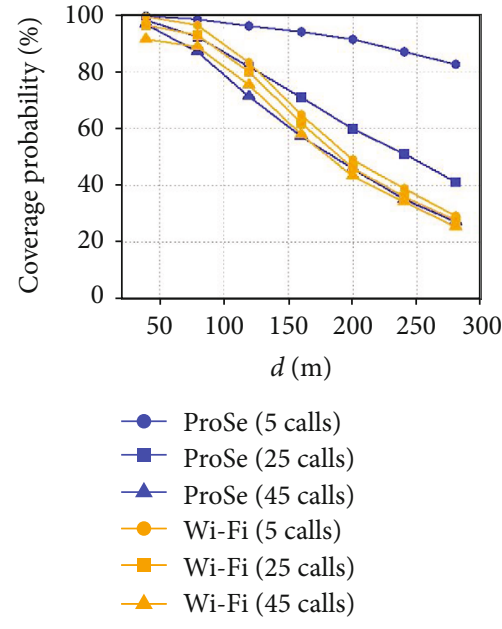

(a)

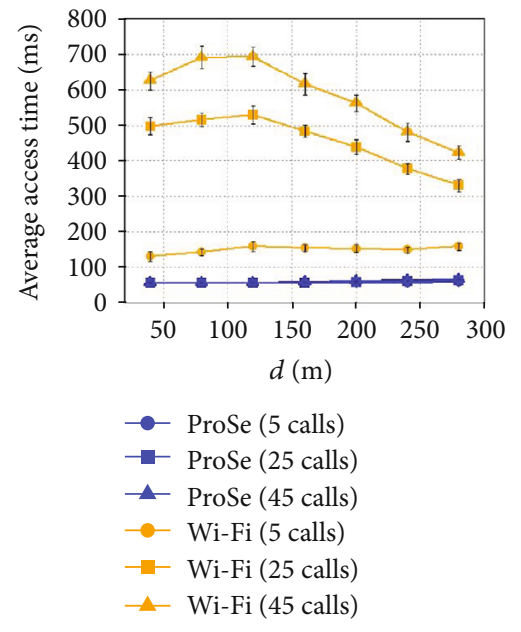

(b)

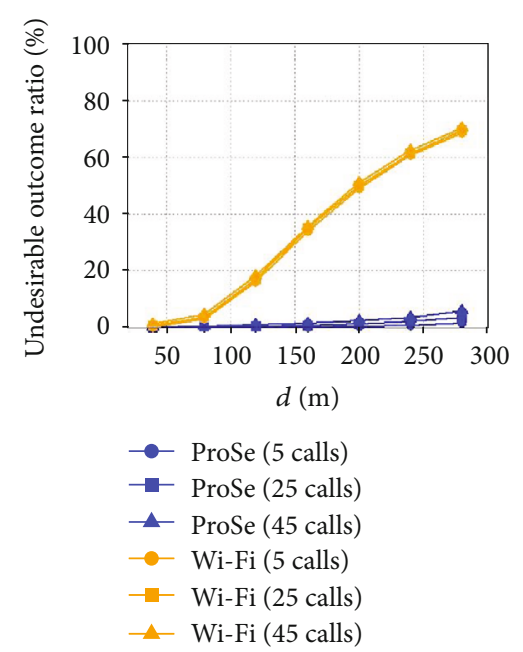

(c)

FIgURE 11: Performance metrics for additional calls using various user densities. (a) User density coverage probability. (b) MCPTT access time. The error bars represent the $95 \%$ confidence interval. (c) Undesirable outcome ratio.

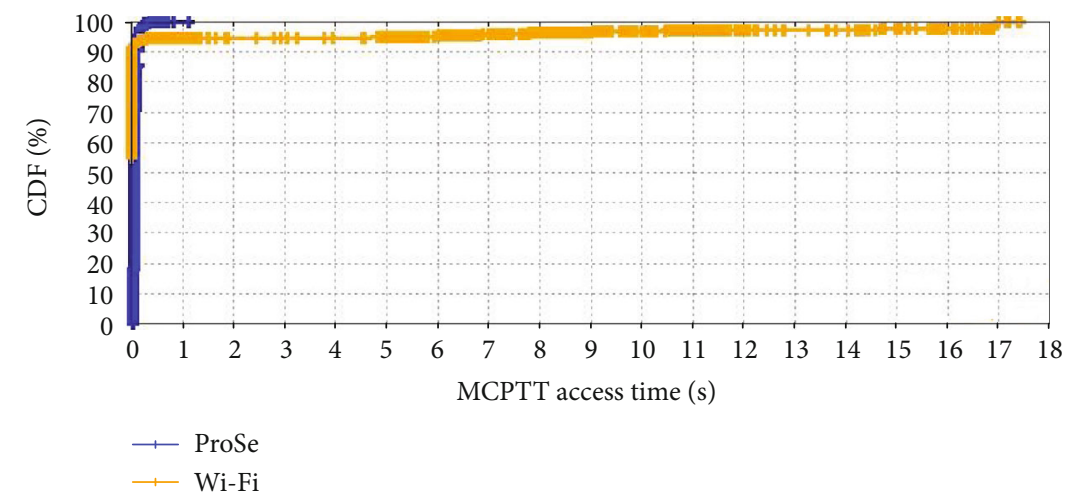

FIgURE 12: CDF of MCPTT access times in the worst cases.

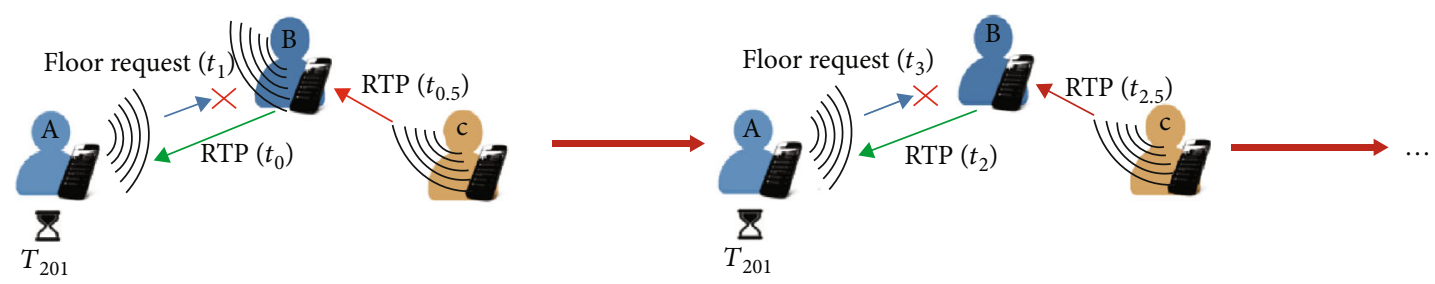

FIGURE 13: Preemptive floor request scenario with RTP and hidden node.

the average access time only increases from $55.61 \mathrm{~ms}$ to $55.64 \mathrm{~ms}$ when the additional calls change from 5 calls to 45 calls.

Figure 11(c) shows that ProSe outperforms Wi-Fi in terms of the ratio of undesirable outcomes, too. For Wi-Fi, the ratio of undesirable outcomes increases from $0.04 \%$ to $1.1 \%$ at $d=40 \mathrm{~m}$ when increasing the number of additional calls from 5 to 45 , and when $d=280 \mathrm{~m}$ and there are 45 additional calls, the ratio reaches $71 \%$. For ProSe, however, the ratio of undesirable outcomes is less than $1 \%$ when $d=40$ $\mathrm{m}$ and there are 45 additional calls and only ever produces a $5.8 \%$ ratio when $d=280 \mathrm{~m}$ with 45 additional calls.

One notable finding from the above analysis is that we observe very large access time values when using $\mathrm{Wi}$-Fi as shown in Figure 12, which captures all of the recorded MCPTT access times using a Cumulative Distribution Function $(\mathrm{CDF})$ in the worst cases. This situation can arise when there are two devices that cannot detect each other's transmissions, as depicted in Figure 13. These "hidden nodes" in combination with the operation of the MCPTT protocol 
make it possible for a floor request to go unanswered indefinitely. Basically, the interaction between the following protocol behaviors made the error scenario possible, assuming $U E_{B}$ is the current floor arbitrator.

(1) $U E_{B}$ is within transmission range of both $U E_{A}$ and $U E_{C}$, but $U E_{A}$ and $U E_{C}$ cannot hear each other due to the hidden node problem. The transmission of $U E_{A}$ and $U E_{C}$ always overlaps so that $U E_{B}$ cannot receive $U E_{A}$ 's floor request message successfully; this is possible because $U E_{A}$ transmits a floor request every $40 \mathrm{~ms}$ and $U E_{C}$ might transmit an RTP packet every $20 \mathrm{~ms}$ even if at the application layer it is not intended for $U E_{B}$; (2) $U E_{B}$ continues sending RTP packets every $20 \mathrm{~ms}$ to $U E_{A}$ since it has not received the floor request from $U E_{A}$ yet; (3) $U E_{A}$ continues transmitting floor request messages and resetting the retransmission timer, $T_{201}$, every $40 \mathrm{~ms}$. $U E_{A}$ 's retransmission counter $C_{201}$ is also reset every time an RTP packet is received from $U E_{B}$, and thus, the upper limit of the retransmission counter $C_{201}$ is not reached.

Therefore, with the combination of (1), (2), and (3), $U E_{A}$ will continue requesting the floor indefinitely and drive the access time to a very large value. This extreme case is more likely to occur over Wi-Fi than over ProSe because (1) is more likely to occur with Wi-Fi because of the different medium access control protocols. When both $U E_{A}$ and $U E_{C}$ try to access the channel at almost the same time, both of them might initiate transmission immediately if sensing that the channel is idle, which leads to a collision and the continued attempts of almost synchronized transmissions, while the hidden node situation keeps the back-offs from taking effect. At this point, only some outside factor such as the user releasing the PTT button (which occurs in our simulation after $20 \mathrm{~s}$ ), the call ending, or a back-off being triggered due to another UE's signal could break the livelock. For ProSe though, the radio resources used for $U E_{A}$ and $U E_{C}$ will be randomized even if they have the packets ready at almost the same time. Therefore, the chances that (1) is true for a ProSe UE are much smaller than for Wi-Fi.

The above observation suggests that if the MCPTT protocol is to be used over Wi-Fi, then it needs to be modified to prevent such a livelock. The solution could be as simple as adding a limit to how long a request can be ongoing or making it possible for the values for the retransmission timers to be variable/randomized. Therefore, the average access time shown for Wi-Fi in Figure 11(b) is an underestimate of its true access time, if the floor request message can be retransmitted indefinitely. On the other hand, for ProSe, we can see that in the worst case, when $d=280 \mathrm{~m}$ and there are 45 additional calls, the average access time is $67.32 \mathrm{~ms}$, the ratio of undesirable outcomes is $6.3 \%$, and the maximum access time experienced is $1.12 \mathrm{~s}$, which may still be reasonable.

\section{Conclusion}

We compare several performance metrics for MCPTT over ProSe vs. MCPTT over Wi-Fi, since ProSe and Wi-Fi are the potential broadband wireless access technologies that first responders may utilize in rural and remote areas when the traditional in-network coverage is not available or desired. Results in Section 3 showed that Wi-Fi may provide faster access times than ProSe when a user wishes to speak when the traffic is relatively light (i.e., less active calls). However, once the traffic was heavier, ProSe outperformed Wi-Fi until the channel was too saturated, at which point the two technologies offered very similar performance. Section 4 showed that with ProSe, better coverage can be achieved at greater distances than $\mathrm{Wi}-\mathrm{Fi}$, which was reflected through a higher MCV coverage probability. Under our simulation assumptions, a $90 \% \mathrm{MCV}$ coverage probability can be achieved anywhere from $305 \mathrm{~m}$ to $530 \mathrm{~m}$ with ProSe, while with $\mathrm{Wi}-\mathrm{Fi}$ the range can be anywhere from $20 \mathrm{~m}$ to $55 \mathrm{~m}$. The variation in range within each technology is attributed to system's configuration. The analysis in Section 5 sought to combine the aspects of Sections 3 and 4 to provide a more comprehensive study. We could see that for a given area, both ProSe and Wi-Fi suffer as the number of transmitting UEs increases and becomes more dense, while ProSe is more susceptible to interference and Wi-Fi lacks coverage range. We also observed very large access times due to the operation of the MCPTT protocol and that modifications may be needed to address this, especially when deploying MCPTT over Wi-Fi.

\section{Data Availability}

The data used to support the findings of this study are available from the corresponding author upon request.

\section{Conflicts of Interest}

The authors declare that they have no conflicts of interest.

\section{References}

[1] 3GPP, Technical Specification Group Radio Access Network, Evolved universal terrestrial radio access (E-UTRA) and evolved universal terrestrial radio access network (E-UTRAN); Overall description; Stage 2 (Release 15); TS 36.300, Technical Specification, 2019.

[2] IEEE, IEEE standard for information technology-telecommunications and information exchange between systems local and metropolitan area networks-specific requirements; IEE 802.11g-2003 Wireless LAN Medium Access Control (MAC) and Physical Layer (PHY), Specifications, Technical Specification, 2003.

[3] G. Baldini, S. Karanasios, D. Allen, and F. Vergari, "Survey of wireless communication technologies for public safety," IEEE Communications Surveys \& Tutorials, vol. 16, no. 2, pp. 619641, 2014.

[4] J. Wang and R. A. Rouil, "BLER performance evaluation of LTE device-to-device communications," NIST IR, vol. 8157, 2016.

[5] J. Wang and R. A. Rouil, "Assessing coverage and throughput for D2D communication," in 2018 IEEE International Conference on Communications (ICC), pp. 1-6, Kansas City, MO, USA, 2018. 
[6] D. Griffith, F. Cintr'on, A. Galazka, T. Hall, and R. Rouil, "Modeling and simulation analysis of the physical sidelink shared channel (PSSCH)," in 2018 IEEE International Conference on Communications (ICC), pp. 1-7, Kansas City, MO, USA, 2018.

[7] Y. Sun, W. Garey, R. Rouil, and P. Varin, "Access time analysis of MCPTT off-network mode over LTE," Wireless Communications and Mobile Computing, vol. 2019, Article ID 2729370, 19 pages, 2019.

[8] R. A. Rouil, F. J. Cintr'on, A. B. Mosbah, and S. M. G. Quintiliani, "Implementation and validation of an LTE D2D model for Ns-3," in Proceedings of the Workshop on ns-3 - 2017 WNS3, pp. 55-62, 2017.

[9] A. Yarali, B. Ahsant, and S. Rahman, "Wireless mesh networking: a key solution for emergency \& rural applications," in 2009 Second International Conference on Advances in Mesh Networks, pp. 143-149, Athens, Glyfada, Greece, 2009.

[10] M. A. R. Siddique, J. Kamruzzaman, and M. J. Hossain, “An Analytical approach for voice capacity estimation over WiFi network using ITU-T E-model," IEEE Transactions on Multimedia, vol. 16, no. 2, pp. 360-372, 2014.

[11] E. Ferro and F. Potorti, "Bluetooth and Wi-Fi wireless protocols: a survey and a comparison," IEEE Wireless Communications, vol. 12, no. 1, pp. 12-26, 2005.

[12] A. B. Fadhah, A. Al-Lawati, S. Al-Maskari, A. Touzene, and A. Al-Kindi, "Experimental performance evaluation of Wireless 802.11 b networks," in International Conference on Applications of Digital Information and Web Technologies, pp. 151-155, Ostrava, Czech Republic, 2008.

[13] G. Pei and T. R. Henderson, Validation of OFDM Error Rate Model in ns-3, Boeing Research \& Technology, 2010, https:// www.nsnam.org/ pei/80211 ofdm.pdf.

[14] G. Anastasi, E. Borgia, M. Conti, and E. Gregori, "IEEE 802.11 ad hoc networks: performance measurements," in 23rd International Conference on Distributed Computing Systems Workshops, pp. 758-763, 2003.

[15] G. Anastasi, E. Borgia, M. Conti, and E. Gregori, "Wi-fi in ad hoc mode: a measurement study," in Second IEEE Annual Conference on Pervasive Computing and Communications, pp. 145-154, Orlando, FL, USA, USA, 2004.

[16] ITU-T, Wideband coding of speech at around $16 \mathrm{kbit} / \mathrm{s}$ using adaptive multi-rate wideband ( $A M R-W B)$, Recommendation, 2003.

[17] 3GPP, Technical Specification Group Core Network and Terminals, Mission critical push to talk (MCPTT) media plane control; Protocol specification (Release 14); TS 24.380, Technical Specification, 2018.

[18] 3GPP, Technical Specification Group Core Network and Terminals, Mission critical push to talk (MCPTT) call control; Protocol specification (Release 14); TS 24.379, Technical Specification, 2018

[19] H. T. Friis, "A note on a simple transmission formula," Proceedings of the IRE, vol. 34, no. 5, pp. 254-256, 1946.

[20] 3GPP, Technical Specification Group Radio Access Network, Study on LTE device to device proximity services; Radio Aspects; TR 36.843, Technical Report, 2015.

[21] IEEE, IEEE standard for information technology-telecommunications and information exchange between systems local and metropolitan area networks-specific requirements; IEEE 802.11 Wireless LAN Medium Access Control (MAC) and Physical Layer (PHY), Specifications, Technical Specification, 2016.
[22] 3GPP, Technical Specification Group Services and System Aspects, Mission critical push to talk (MCPTT); TS 22.179, Technical Specification, 2018.

[23] 3GPP, Technical Specification Group Services and System Aspects, Policy and charging control architecture; TS 23.203 (Release 14), Technical Specification, 2018.

[24] 3GPP, Technical Specification Group Radio Access Network, Evolved universal terrestrial radio access (E-UTRA); Physical Layer Procedures; TS 36.213, Technical Specification, 2018. 